\title{
Internet of Things (IoT) Based Smart Environment Integrating Various Business Applications and Recent Research Directions
}

\author{
Dr. Vijeta Kumawat ${ }^{1}$, B. Umamaheswari ${ }^{2}$ \\ ${ }^{1}$ Associate Professor, ${ }^{2}$ Assistant Professor \\ 1, 2JECRC College of Engineering, Jaipur, Rajasthan, India
}

\begin{abstract}
How to cite this paper: Dr. Vijeta Kumawat | B. Umamaheswari "Internet of Things (IoT) Based Smart Environment Integrating Various Business Applications and Recent Research Directions" Published in International Journal of Trend in Scientific Research and Development (ijtsrd), ISSN: 24566470, Volume-3 | Issue-4, June 2019, pp.422-425, URL: https://www.ijtsrd.c om/papers/ijtsrd23 658.pdf

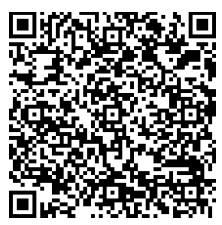
IITSRD23658
\end{abstract}

Copyright (C) 2019 by author(s) and International Journal of Trend in Scientific Research and Development Journal. This is an Open Access article distributed under the terms of the Creative Commons

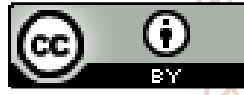
Attribution License (CC BY 4.0) (http://creativecommons.org/licenses/ by $/ 4.0$ )

\section{APPLICATIONS}

Recently, the IoT technology has appeared as a one of the basics in our lives because it uses almost every areas such healthcare, smart water, agriculture, transportations, surveillance and so on. Furthermore, there are many applications which use this concept.

Healthcare: the IoT Cloud paradigm has been broadly used in the healthcare region to diagnosis, treatment and tracking the status of the patients at all. The paradigm ought to serve four pivots namely, i) tracking: it is a purpose aimed to identify the patient in motion, ii) identification and authentication: identification aimed to reduce mistakes in diagnosis and authentication used to meet with security requirements, iii) data collection: It is related to integrating RFID technology it's typically aims to reduce processing time and finally iv) sensing: it is used to provide real time information about the patient.

Smart environment: To create the smart environment such as smart city or smart home while maintaining the level of service without degradation has relied on the integration between both IoT and cloud computing. The Smart Home technology ranking as the highest Internet of Things application on all measured channels. This includes famous startup names such as Nest or AlertMe in addition to a number of multinational corporations like Philips, Haier, or Belkin. Smart city span a wide variety of use cases, from traffic management to water distribution, waste management, urban security and environmental monitoring. Its fame is fueled by the fact that many Smart City solutions promise to ease real pains of people living in cities these days. IoT solutions in the area of Smart City explain traffic congestion problems, decrease noise and pollution and help make cities safer.

Normally, The IoT suffers from a host of the thorniest issues, first of all, heterogeneity of objects; there are many middleware technologies are designed to deal with this type of issues, such as RFID middleware and WSN middleware. On the other hand, the cloud computing provides scalability and hide complexity of sensors from end users by using the virtualization technology. The most popular challenges in this type of application are limited to security and real-time applications.Video surveillance: is an intelligent video to monitor the object behavior and activities, it has become an important tool of security-related application and it can be considered as an alternative to self-contained management systems, and the complex video analytics requires Cloudbased solutions, and it can be used to satisfy the need to increase volume of storage capacity of media. 


\section{Smart Farming}

Smart farming is an often disregarded business case for the IoT because it does not really fit into the well known category such as industries, health and mobility. on the other hand, due to the remoteness of farming operations and the large number of livestock that could be monitored the Internet of Things could revolutionize the way farmers work. But this thought has not yet reached large scale attention. Nevertheless, one of the IoT applications that should not be underestimated. Smart farming will become the important application field in the mostly agricultural product exporting countries.

Automotive and smart mobility: the aim of this function intense on the improvement of transportation and transport during increasing safety road, reducing congestion, and manage traffic. Can be considered the integration between Cloud Computing and IoT as a promising opportunity to find efficient solution is characterized by high performance, security and lowest cost. The challenges in this type are identification, heterogeneity of sensors, scalability and dynamic behavior of objects.

Smart energy and smart grid: the biggest difficulty in the sensors world is power consumption, the battery in sensors may exhaust very quickly, and both the IoT and Cloud Computing provide intelligent solutions to manage energy distribution and consumption in heterogeneous environments.

\section{ARCHITECTURE FOR INTEGRATED IOT BASED SMART ENVIRONMENT}

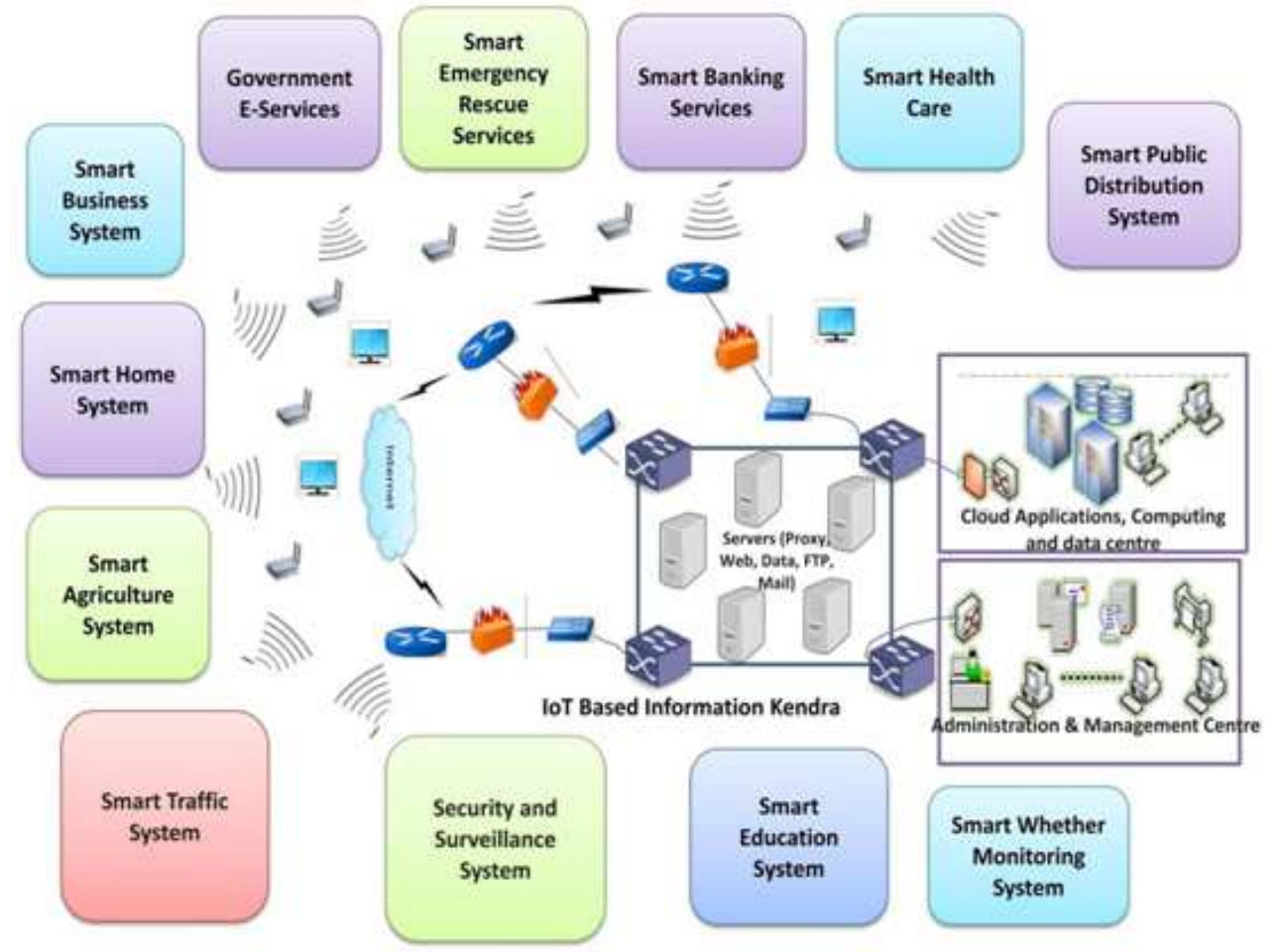

Fig-1: Architecture for IoT based Smart Environment

This section of the paper describes the overall architecture for the smart environment. Also discussed a layered framework for IoT based smart environment. The functioning of RFID system is explicated. The different possible business applications and sectors to form the smart environment as well are deal.

Layered Framework for IoT based smart environment The layered framework for IoT based smart environment consists of four layers as shown in figure 3. They are device layer, middleware layer, network layer and application layer. Each layer is described below in short.

\subsubsection{Perception layer}

Perception layer is also recognized as device layer. All the sensor devices, RFID tags and various other physical objects go to this layer. This layer is supported by the wireless sensor network, RFID antennas and readers. The sensor devices and RFID tags in the layer help to gather specific information associated to the objects in the dissimilar business domains. The collected information may be location, temperature, sound, pressure, heart pulse, humidity, climatic condition and so on. The incidental data is sent further to middleware layer.

Application layer

(Smart Services and Applications of different domain)

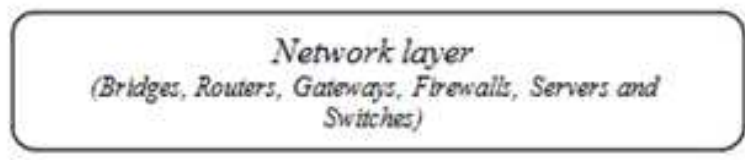

Data Conversion layer

(Middle ware)

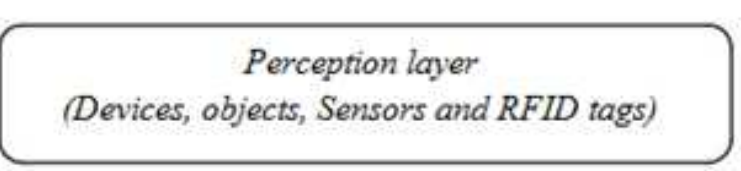

Figure3: Layered Framework for IoT based 


\subsubsection{Data Conversion Layer}

There may be numerous heterogeneous signals from sensors and RFID tags in the tiny devices and objects attached to business applications integrated in the smart environment. The data collected in the form of heterogeneous signals cannot be sent to the conventional network for further processing of data. Majority of the signals might be in analogous form. Thus, this layer is responsible for converting special signals inferred into data that might be sent through the conventional network. This layer consists of middleware which wires the conversion task.

\subsubsection{Network Layer}

Network Layer is as well recognized as transport layer which is responsible for fetching the data to IoT information kendra for processing. The decision making method based the on the data sent will be supported with cloud applications data centre and an administrative management centre. This layer is functions based on TCP/IP as it dishonesty on conventional network. It consists of gateways, routers, switches, firewalls and servers.

\subsubsection{Application Layer}

The application layer is comprised of all the business applications in the smart environment planned. The implementation of each business sector differs by their applications and services. This layer is in charge for the decision making process based the data inferred from the objects and devices attached to the business domains.

\subsection{Radio frequency Identification (RFID)}

RFID means Radio Frequency Identification. It basically used to recognize objects and people using radio technology of short range. These objects and people as well can communicate with each other digitally. There are 2 entities here: Reader which is stationary and Tag which is movable. This tag on any object is being read by the reader to identify it. Readers have their own battery supply and instantiate the communication. RFID uses Ultra wide Bandwidth (UWB) technology to enhance RFID performance in a specific IoT application field. RFID systems consist of three components in two combinations: a transceiver and antenna are frequently combined as an RFID reader.

RFID is a get through in embedded communication and WSN, RFID is used to make a unique ID for the object in WSN. It consists of two parts are passive RFID: which used to power of the reader's interrogation signal to communicate the ID to the RFID, and access control.

RFID systems and accomplishment is supermarket chains. All the goods are the shopping bag of the customer may be identified by RFID reader as all the products have RFID tags on them. After identifying the products, the information is send to the backend which further provides the additional information such as prices of the products, discounts on the qualifying products and for the qualifying customers, etc. in addition the backend reduces the number of products in the database and notifies the concerned authority if the stock of the products has to be updated

\section{Wireless Sensor Networks (WSN)}

WSN is a wireless network consisting of spatially distributed independent devices using sensors to politely monitor physical or environmental conditions, such as temperature, sound, vibration, pressure, motion or pollutants, at different locations.

There are thousands of wireless sensor nodes which have some computational power, some memory, limited bandwidth and sensing capability. They capture and store the data of the environmental conditions and send the data to a sink or a base station which further processes and analyzes the data. The sensor nodes can communicate with each other using radio signals. Some real time applications of WSN include transportation, military, area monitoring, healthcare etc. WSN is an important part of IoT, it's considered as a core to build the IoT block, it consists of a group of specialized sensor data are shared among sensor nodes with communication infrastructure for monitoring some of events or states of objects such as temperature, sound, pressure, etc. these sensor nodes work autonomously and can be linked between them by self-organizing.

\section{CONCLUSION}

IoT has been regularly bringing a sea of technical changes in our daily lives, which in turn helps to making our life simpler and more contented, though various technologies and applications. There is numerous usefulness of IoT applications into all the domains including medical, manufacturing, industrial, transportation, education, governance, mining, habitat etc.This paper has sought to highlight the IoT concept in general through the three sections namely......... This paper described how IoT could put together the different business domain under smart environment duly supported by IoT Information Kendra. The IoT information Kendra will provide general public with a lot of services if they are established in each district or region. This paper has brought out some feasible applications in every domain. since the IoT based smart environment has huge benefits the number of challenges and issues are many and they have to be addressed properly. The use of this planned IoT based Smart Environment will be difficult but at the same time it has numerous benefits to the society in near future.

\section{References:}

[1] Sarfraz Alam, Mohammad M. R. Chowdhury, Josef Noll, 2010 SenaaS:An Event-driven Sensor Virtualization Approach for Internet of Things Cloud, Networked Embedded Systems for Enterprise Applications (NESEA), 2010 IEEE International Conference on, 1-6.

[2] Andras Kalmar, Rolland Vida, Markosz Maliosz, 2013 Context-aware Addressing in the Internet of Things using Bloom Filters, Cog 1 nfoCom 2013. 4th IEEE I nternational Conference on Cognitive I nfocommun ications' (Dec. 2013) 487 - 492.

[3] Jayavardhana Gubbi, Rajkumar Buyya, Slaven Marusic, Marimuthu Palaniswami, 2013 Internet of Things (IoT): A Vision, Architectural Elements, and Future Directions. Future Generation Computer Systems, 1645-1660.

[4] Daoliang Li, Yingyi Chen, Oct. 2010, Computer and Computing Technologies in Agriculture. Springer, 2431.

[5] L. Atzori, A. lera, G. Morabito, The Internet of Things: Survey. Computer networks, 2787-2805. 
[6] S. Haller, S. Karnouskos, and C. Schroth, "The Internet of Things in an enterprise context," Future Internet Systems (FIS), LCNS, vol. 5468. Springer, 2008, pp. 148.

[7] Anne James, Joshua Cooper, Keith Jeffery, and Gunter

Saake. "Research Directions in Database Architectures for the Internet of Things: A Communication of the First International Workshop on Database Architectures for the internet of things (DAIT 2009)," BNCOD2009: 225233.

[8] Zhang Lin. "School of Management, Zhejiang University, Prof. Liu Yuan: The business scale of communications between smart objects is tens of times the scale of communications between persons," Science Times. 2009.11.16. (in Chinese)

[9] H. Jiawei and M. Kamber, "Data Mining: Concepts and Techniques”, Morgan Kaufmann, 2011.
[10] Dieter Uckelmann, Mark Harrison, Florian Michahelles, 2011. An Architectural Approach Towards the Future Internet of Things. Architecting Internet of Things by Springer,1-22.

[11] Kevin C. Desouza, David Swindell, Kendra L. Smith, Alison Sutherland, Kena Fedorschak, and Carolina Coronel, 2015. Issues in Technologies innovation, (May 2015)

[12] Jayavardhana Gubbi a , Rajkumar Buyya b, Slaven Marusic a , Marimuthu Palaniswami, 2013. Internet of Things (IoT): A vision, architectural elements, and future directions. Future Generation Computer Systems $29,1645-1660$.

[13] Elkhodr, M. Shahrestani, S, Hon Cheung, 2013. The Internet of Things: Vision \& Challenges. TENCON IEEE Spring Conference, 218- 222.

[14] K. Ashton, 2009. That 'Internet of Things' Thing. RFid Journal, 97-114. 